Opinion

\title{
Surfing the wave of urine
}

\section{Opinion}

Urine was thought to reflect human health 6000 years ago. ${ }^{1}$ But why was it almost abandoned compare to blood in current biomarker research? I guess it was considered hopeless as a biomarker source because normally it changes too much all the time. Biomarker researchers apparently enjoy gliding on the straight line of blood rather than surfing on the wave of urine. I thought about there are probably some straight lines in urine too. If there are bumps on those straight lines in urine, they can be good biomarkers. ${ }^{2}$ But urine is not blood. We cannot expect too many straight lines in urine like in blood, because blood is controlled by homeostatic mechanisms in the body to keep the line relatively straight. But what are biomarkers at all and where are most of them? Biomarkers are the measurable changes associated with a physiological or pathophysiological process. ${ }^{3}$ They are changes. They suppose to stay in the place where there are a lot of changes, like urine. Urine changes all the time in healthy individuals means that urine changes with many physiological factors, means it is sensitive enough to reflect the physiological conditions. If urine is sensitive enough to reflect physiological factors, it is not difficult to imagine it must be able to detect the early and subtle changes of pathological processes. What if the pathological changes are too subtle and were buried under the wave of physiological variations? Some of the changes will be. They cannot become biomarkers. But what if some of the changes caused by pathological processes are big enough waves by themselves, which are much higher than the waves of physiological processes? Then they are good biomarkers. Those are the kind of waves biomarker researchers should look for and surf on. ${ }^{4}$ No matter how big the physiological variations are, as long as the changes cause by the pathological process is bigger, they are good biomarkers. Let's surf the wave of urine.

\section{Acknowledgements}

This work was supported by the National Basic Research Program

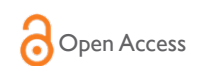

CrossMark

Volume 2 Issue 3 - 2015

\section{Youhe Gao}

Department of Biochemistry and Molecular Biology, Beijing Normal University, China

Correspondence: Youhe Gao, Department of Biochemistry and Molecular Biology, Beijing Normal University, Gene Engineering and Biotechnology Beijing Key Laboratory, Beijing, I00875, P. R. of China, Tel 861058804382,

Email gaoyouhe@bnu.edu.cn

Received: May 19, 2015 | Published: May 26, 2015

of China (2012CB517606, 2013CB530805), the Key Basic Research Program of China (No. 2013FY114100).

\section{Conflict of interest}

The author declares no conflict of interest.

\section{References}

1. Armstrong JA. Urinalysis in Western culture: a brief history. Kidney Int. 2007;71(5):384-387.

2. Sun W, Chen Y, Li F, et al. Dynamic urinary proteomic analysis reveals stable proteins to be potential biomarkers. Proteomics-clinical applications. 2009;3(3):370-382.

3. Gao Y. Urine-an untapped goldmine for biomarker discovery? Sci China Life Sci. 2013;56(12):1145-1146.

4. Gao Y. Opinion: Roadmap to the Urine Biomarker Era. MOJ Proteomics Bioinform. 2014;1(1):1. 